\title{
Optimization of reward rate on concurrent variable-interval variable-interval schedules
}

\author{
S. A. HENSCH and C. DONALD HETH \\ University of Alberta, Edmonton, Alberta, Canada
}

\begin{abstract}
Six rats were placed on concurrent variable-interval variable-interval schedules with a 15 -sec changeover delay (COD). The variable-interval schedules were varied such that the COD comprised between $25 \%$ and $100 \%$ of the average interreinforcement interval of the more favorable alternative. The obtained reinforcement rate and the rate of changing from one schedule to the other were compared to predictions of Houston and McNamara's (1981) optimality model of concurrent choice. The pattern of behavioral allocation was consistent with the predictions of the model, although none of the animals was able to achieve optimal performance on any of the presented schedules. Observed behavior reliably tracked optimal behavior in that the ratio of obtained reinforcers to the optimum predicted by Houston and McNamara did not vary as the underlying schedule parameters was changed.
\end{abstract}

The behavior of animals responding under concurrent variable-interval variable-interval (conc VI VI) schedules of positive reinforcement has been described in various ways. Findley (1958) reported that preference for a given schedule of a pair of concurrently available independent schedules varied inversely with the mean of the interreinforcement interval (IRI). Herrnstein (1961) suggested a quantitative steady-state description, now known as the matching law, in which relative preference for one schedule was related to the relative rate of reinforcement obtained from that schedule:

$$
B_{1} /\left(B_{1}+B_{2}\right)=r_{1} /\left(r_{1}+r_{2}\right),
$$

where $B_{i}$ is a measure of responses made to, or of time spent on, schedule $i$, and $r_{i}$ is the rate with which reinforcers are earned on schedule $i$. Baum (1974), in an influential review, suggested a generalization of this rule, using a power function. Empirical studies have found that the matching law provides an adequate description of concurrent performance (see, e.g., Baum, 1979; Wearden \& Burgess, 1982).

An alternative approach to the description of conc VI VI performance is to view the concurrent schedule procedure as a choice paradigm, and to derive the animal's behavior from some set of assumptions regarding choice. One such analysis was developed by Herrnstein and Vaughan (1980) on the basis of the principle of "melio-

Portions of this study were submitted by S. A. Hensch to the University of Alberta in partial fulfillment of the requirements for the degree of master of science, and were reported at the annual meeting of the Canadian Psychological Association, Montréal, Québec, 1988. This research was supported in part by a grant from the Natural Sciences and Engineering Research Council of Canada to C. D. Heth. We thank W. F. Epling and W. D. Pierce for the use of their laboratory facilities, and acknowledge the helpful suggestions and comments provided to us by the reviewers. Requests for reprints may be addressed to either author at the Department of Psychology, University of Alberta, Edmonton, Alberta T6G 2E9, Canada. ration." They defined melioration as behavioral allocation that equalizes the local rates of reinforcement on the alternatives. Specifically, animals are assumed to minimize the difference:

$$
R_{D}=\left(R_{1} / t_{1}\right)-\left(R_{2} / t_{2}\right),
$$

where $R_{D}$ is the difference in the local rates of reinforcement, $R_{i}$ is the number of reinforcers obtained on schedule $i$, and $t_{i}$ is the time spent on schedule $i$.

Houston and McNamara (1981) also developed an analysis of conc VI VI behavior based on axioms of choice. Basically, they viewed the animal as selecting a particular response strategy so as to maximixe the overall rate of reinforcement, $\boldsymbol{R}_{\boldsymbol{O}}$. Using certain simplifying assumptions concerning the conc VI VI procedure, they first derived the relationship between expected reinforcement and the time spent responding to an alternative. They showed that expected reinforcement on alternative $i$ is equal to

$$
R_{i}=1-\exp \left[\left(-\lambda_{i}\left(a_{j}+\tau_{i}+\tau_{j}\right)\right]+\lambda_{i} a_{i},\right.
$$

where $R_{i}$ is the expected number of reinforcers each time an animal completes a cycle of leaving alternative $i$, spending time on $j$, and returning to, and responding on, $i ; \lambda_{i}$ is the rate of reinforcement on alternative $i ; a_{i}$ and $a_{j}$ are the times spent on each alternative after the changeover delay (COD) has elapsed; and $\tau_{i}$ and $\tau_{j}$ are the lengths of the COD for the two alternatives. Houston and McNamara then derived the relationship between $R_{o}$ (the sum over all the alternatives $\boldsymbol{R}_{i}$ ) and the time spent on either schedule before switching to the other. The optimal value of $\boldsymbol{R}_{O}$ is specified by differentiating $\boldsymbol{R}_{o}$ with respect to these two times and setting the results equal to zero. According to this analysis, the optimal value is one at which

$$
R_{o}=1+\lambda \exp \left[-\lambda\left(a_{1}+2 \tau\right)\right]
$$

and 


$$
R_{o}=\lambda+\exp \left[-\left(a_{2}+2 \tau\right)\right],
$$

where $a_{1}$ and $a_{2}$ are the times spent on each schedule after the COD has elapsed, $\tau$ is the ratio of the COD to the richer schedule, and $\lambda$ is the ratio of the richer schedule to the leaner schedule. The COD is a programmed delay that separates a reinforcer on one alternative from the first response to that alternative.

Intuitively, Houston and McNamara's (1981) model portrays the COD as the cost of leaving one schedule to go to another. For example, an animal switching rapidly between conc VI 1-min VI 1-min schedules, with no COD contingency, could obtain an average of one reinforcer every $30 \mathrm{sec}$. However, if the animal continues to switch rapidly when a COD contingency is introduced, these switches will cost the animal in terms of a period of time during which no reinforcers are available, decreasing the maximum number of reinforcers available within a session. In the conc VI 1-min VI 1-min example, any COD reduces the average number of reinforcers that can be obtained. In general, the animal exchanges the time of the COD for the reinforcements obtained from the new schedule. The optimal tradeoff is specified by Equations 4 and 5.

Houston and McNamara (1981) considered the solution of Equations 4 and 5 within the context of three different response strategies: (1) never switch, wherein the animal never leaves the schedule with the higher reward rate; (2) stay-stay, wherein the animal stays for some positive length of time on each schedule before switching away to the alternative; and (3) stay-switch, wherein the animal switches into the leaner schedule only to collect a reinforcer, and then immediately switches back to the richer schedule. Houston and McNamara predicted that the particular strategy exhibited by an animal will be determined by the two parameters, $\tau$ and $\lambda$, relative to two constants, $\tau_{c}$ (approximately equal to .92) and $\lambda_{c}$ (approximately equal to .84 ).

The first constant $\left(\tau_{c}\right)$ corresponds to cases in which the COD is approximately $92 \%$ of the average IRI on the richer of two concurrently available schedules. The second constant $\left(\lambda_{c}\right)$ corresponds to cases in which the average IRI on the richer of two schedules is approximately $84 \%$ of the average IRI on the leaner schedule. When $\lambda$ is less than .84 , the optimal strategy is either never switch or stay-switch, depending on the value of $\tau$. When $\tau$ is greater than .92 and $\lambda$ is less than .84 , never switch is the optimal strategy; when $\tau$ is less than .92 and $\lambda$ is less than .84 , stay-switch is the strategy that will optimize the reward rate.

Consider a conc VI 1-min VI 2-min $(\lambda<$.84) schedule. If the COD were $1-\mathrm{min}$ long $(\tau>.92)$, then each switch from the VI 1-min schedule to the VI 2-min schedule would cost the animal a 1-min period without reinforcement. Under the assumptions of the model, this cost would be greater than the benefits derived from switching; therefore, the animal should never switch away from the better schedule. However, if the COD were $30 \mathrm{sec}$ $(\tau<.92)$, switching to the VI 2-min schedule after a sufficient amount of time on the VI 1-min schedule would sometimes produce a reinforcer immediately after the 30-sec COD. According to the model, this benefit would exceed the cost of switching; therefore, the animal should exhibit a stay-switch response pattern.

As $\lambda$ increases beyond .84 (i.e., as the average IRIs on the two schedules approach equality) the never switch and stay-switch regions give way to a region in which stay-stay becomes the optimal strategy. This strategy is characterized by staying on each of the schedules for some specified length of time after the COD has timed out. When $\lambda$ is equal to 1.0 , the underlying schedules are equivalent and, consequently, the optimal stay times for each alternative will also be equal. The specific strategy for any given value of $\lambda$ greater than .84 is dependent on the value of $\tau$. As $\tau$ increases from 0 to .92 (i.e., increases from no COD to a COD that is $92 \%$ of the average IRI on the richer schedule), the optimal strategy changes from stay-switch to stay-stay. This means that rather than switching away from the leaner alternative as soon as the COD has timed out, the optimal strategy becomes one of staying for some time beyond the COD on each alternative. Alternatively, as $\tau$ decreases from 1.0 to .92 (for $\lambda>$.84), the optimal strategy changes from one of never switching from the richer of the two alternatives to one of staying on each alternative for some time after the COD has timed out. (For a mathematical derivation of the stay-stay region see Houston \& McNamara, 1981, pp. 373-375). Therefore, Houston and McNamara treated the COD as one of the independent variables, unlike previous treatments that viewed the COD only as a necessary precondition for matching (see, e.g., Allison \& Lloyd, 1971; de Villiers, 1977; Norman \& McSweeney, 1978).

Houston and McNamara's (1981) model provides a useful comparison both to the matching law's and to melioration's description of conc VI VI performance. Under the matching law (Equation 1), it is the ratio of rates of responding and reinforcement that is described; the specific response and reinforcement rates that comprise the ratios are not uniquely specified by the law. Similarly, Herrnstein and Vaughan's (1980) melioration model (Equation 2) does not yield a unique solution. For example, Equation 3 can be used to specify the value of $\boldsymbol{R}_{\boldsymbol{D}}$ by computing the expected number of reinforcers, $R_{i}$, and dividing $R_{i}$ by $a_{i}+\tau_{i}$. Consider the case in which alternatives $i$ and $j$ consist of 30- and 60-sec VIs, respectively, and both CODS are $15 \mathrm{sec}$. Spending $5 \mathrm{sec}$ on alternative $j$ and $34.62 \mathrm{sec}$ on alternative $i$ (after the COD has elapsed) satisfies the melioration condition. However, the condition can also be satisfied by spending $10 \mathrm{sec}$ on alternative $j$ and $53.80 \mathrm{sec}$ on alternative $i$, by spending $15 \mathrm{sec}$ on alternative $j$ and $79.25 \mathrm{sec}$ on alternative $i$, and so on.

In contrast, the model developed by Houston and McNamara (1981) makes specific predictions with respect to the time allocation between the two schedules that will result in optimal performance as a function of $\tau$ and $\lambda$. 
It is therefore of interest to determine whether the more specific model is empirically supported.

As noted by Houston and McNamara (1981), the usual parameters of a concurrent procedure (with a COD that is short relative to the reinforcement schedules) generate predictions within the stay-switch and stay-stay regions. Within these regions, Houston and McNamara found qualitative support for their model. Specifically, they examined the rate with which animals switched from one schedule to the other (the switch rate, or $S$ ). The optimal value of $S$ is determined by $\tau$ and the optimal value of $a_{1}$, and can be calculated with Equation 4. Houston and McNamara's model predicts that the changeover rate will decline as the COD is increased, for a constant pair of concurrent schedules. The model also predicts that for a constant COD, the changeover rate will increase as the average IRIs on the two schedules approach equality. Both trends have been noted in the literature (e.g., de Villiers, 1977).

The present study was designed to determine the effects on concurrent choice behavior when $\tau$ ranges between .25 and 1.00. Four schedule pairs and a COD were selected with values of $\tau$ and $\lambda$ that would predict patterns of behavior within each of the regions described by Houston and McNamara (1981). In one condition, $\tau$ was set to a value of 1.00; using Houston and McNamara's model, it was hypothesized that animals would adopt a never switch strategy and would show exclusive preference for the richer schedule. In two conditions, $\lambda$ was set equal to .50 and $\tau$ was set to either .25 or .50 . In these conditions, it was predicted that animals would exhibit a stay-switch strategy and would spend most of their time on the richer schedule, switching to the leaner schedule only to collect a reinforcer. In a fourth condition, $\lambda$ was set equal to 1.00 and $\tau$ was set equal to .25 (the schedules in the concurrent pair were equivalent and the COD was one fourth of the average IRI). It was hypothesized that under these conditions, animals would show a stay-stay strategy, with an equal distribution of behavior across the two alternatives. The animals' obtained reward rates and observed switch rates were compared to the optimal rates predicted by the model under each of these conditions.

\section{METHOD}

\section{Subjects}

Six experimentally naive male Long Evans rats, approximately 12 weeks old at the start of training, were used. The rats were housed individually under a constant cycle of $12 \mathrm{~h}$ light and $12 \mathrm{~h}$ dark. The rats were fed at the end of each session, and were maintained at approximately $80 \%$ of their extrapolated normal weight, which was calculated by allowing a 5-g/week weight gain until the subjects were 16 weeks old. Tap water was freely available at all times in both the home cage and the operant chamber.

\section{Apparatus}

An operant chamber measuring $40 \times 20 \times 20 \mathrm{~cm}$ was constructed. A food cup was located $4 \mathrm{~cm}$ from the floor in the center of the front wall. Directly above the food cup, $6 \mathrm{~cm}$ from the floor, was a feeder tube connected to a dispenser that delivered 45-mg Noyes pellets as reinforcement for bar pressing. Above the food cup, $10 \mathrm{~cm}$ from the floor, was a drinking tube. Levers were situated $6 \mathrm{~cm}$ from the floor in each of the end walls. The chamber was enclosed by sound-attenuating material. House lights remained on during each session. A microcomputer, located in an adjoining room, was used for programming and recording.

\section{Procedure}

After being reduced to $80 \%$ of their ad-lib weight, all subjects were trained to lever press in the operant chamber described above. During the first session, the subjects were placed in the chamber and 40 Noyes pellets were delivered on a fixed-time (FT) $30-\mathrm{sec}$ schedule. During this initial session, the FT 30 -sec schedule could be overridden by a single lever press on either of the available levers. During the next 2 to 6 days, all subjects were given daily sessions utilizing concurrent continuous reinforcement, continuous reinforcement schedules (conc CRF CRF). These sessions ran for $45 \mathrm{~min}$, or until $\mathbf{4 0}$ reinforcers had been delivered, whichever occurred first. Subjects 2 and 5 required additional hand shaping. When each subject was able to complete a 40-reinforcer session in less than $15 \mathrm{~min}$, the following sessions were conducted to ensure that conditioning to both bars had occurred: (1) 40 Noyes pellets were delivered on a conc CRF CRF schedule with the previously preferred lever removed from the chamber to prevent responding; (2) 40 Noyes pellets were delivered on a conc CRF CRF schedule with both levers in place; (3) 40 Noyes pellets were delivered on a conc CRF CRF with the previously nonpreferred lever removed from the chamber; and (4) 40 Noyes pellets were delivered on a conc CRF CRF schedule with both levers in place.

Four additional sessions were run, utilizing a conc FR5 FR5 schedule, during which the session length was extended until 80 reinforcers had been delivered. At this point, the subjects began a series of conc VI VI schedules containing a 15-sec COD. The COD was programmed in such a way that the initial response following a change from one schedule to the other initiated a 15-sec period during which no reinforcers would be delivered. Perseverative responding to an alternative during the COD had no effect on the duration of the COD; however, these responses accumulated and were included in the total number of responses for each session. If a reinforcer had already been set up on an alternative, or if a reinforcer was set up during the $C O D$, it was held; the first response after the 15 -sec COD had elapsed produced the reinforcer and restarted the VI timer associated with that alternative. If the subject switched back to the initial lever before the COD had elapsed, the 15-sec COD timer was reset and the COD began anew. In this way, $15 \mathrm{sec}$ was the minimum period of nonreinforcement following any switch between the two concurrently available alternatives.

The schedules and the number of days for each subject to meet the stability criterion are presented in Table 1 . Stability was achieved when the response rates on both schedules fell within the previous range for 5 consecutive days (Norman \& McSweeney, 1978, p. 455).

The main part of the experiment consisted of the presentation of five separate pairs of conc VI VI schedules. The first pair presented

Table 1

Number of Sessions on Each Concurrent Schedule by Subject

\begin{tabular}{cccccc}
\hline & \multicolumn{5}{c}{ Alternate Schedule Value } \\
\cline { 2 - 6 } Subject & 1 min & 15 sec & 30 sec & 2 min & 1 min \\
\hline 1 & 16 & 25 & 16 & 12 & 17 \\
2 & 36 & 17 & 13 & 11 & 33 \\
3 & 32 & 13 & 31 & 15 & 17 \\
4 & 25 & 17 & 26 & 18 & 17 \\
5 & 12 & 20 & 13 & 20 & 16 \\
6 & 21 & 41 & 24 & 28 & 11 \\
\hline
\end{tabular}


was conc VI 1-min VI 1-min. After stability was achieved on this pair, the second set of schedules (conc VI 15-sec VI 1-min) were presented.

For half of the subjects $(1,3$, and 5) the VI 15-sec component was associated with the right lever, as the subject faced the food cup; for the remaining subjects $(2,4$, and 6$)$ the left lever was used. During the third phase of the experiment (conc VI 30-sec VI 1-min), the richer schedule was presented on the left lever for Subjects 1, 3 , and 5, and on the right lever for Subjects 2, 4, and 6. The leaner schedule of the fourth set of schedules (conc VI 1-min VI 2-min) was presented on the same lever as the VI 30-sec schedule of the previous pair for all subjects. In the final phase, conc VI 1-min VI 1-min schedules were repeated. This presentation ordering was undertaken to control for hysteresis effects (Baum, 1974; de Villiers, 1977; Stevens, 1957) that have been found when the richer schedule is always programmed on the same lever or key.

For all schedules, the IRIs were constructed as a 12-interval series as described by Catania and Reynolds (1968, p. 381). Each completion of the block of 12 intervals initiated a new random presentation of the series. Sessions took place daily, and were terminated when 81 reinforcers had been delivered, or when $60 \mathrm{~min}$ had elapsed, whichever occurred first. Cumulative records from all conditions were randomly checked for any signs of satiation during the experimental session. No decrease in overall response rates across the sessions was detected for any of the animals. Sessions started at $0530 \mathrm{~h}$ MST, 7 days per week. The running order for individual subjects was randomized within the daily time block.

\section{RESULTS}

The number of responses made to each side, the number of seconds spent on that side, and the number of reinforcers earned were averaged over the last 5 days of each condition for each subject. These data are presented in Table 2. The number of responses includes all responses emitted on a given lever during a session. The measure of time spent on a given lever during each session is the cumulative total of the elapsed time from the first response on that lever to the first response on the other lever. In all cases, responding was rapid and continuous throughout the sessions with overall average response rates of $73.09,43.09,84.92,61.64,32.16$, and 42.06 responses per minute for Subjects 1 through 6, respectively. In addition, visual inspection of the cumulative records from the last five sessions of each condition for each subject indicated that the subjects responded throughout the COD; calculation of the average stay time following a switch indicated that in all but the VI 15-sec VI 1-min condition, the subjects tended to stay throughout the COD once a switch had occurred. In the conc VI 15-sec VI 1-min and the conc VI 30-sec VI 1-min conditions, all sessions were terminated after 81 reinforcers were delivered. In the conc VI 1-min VI 1-min and the conc VI 1-min VI 2-min conditions, the sessions were terminated after $60 \mathrm{~min}$ had elapsed. As a result, the subjects received less overall reinforcement in the latter two conditions across the five stability sessions.

The data from Table 2 were initially evaluated with respect to the proportional matching law (Herrnstein, 1961). Proportional time and response measures were calculated, and each was regressed on obtained reinforcement proportions. The $r^{2}$ values associated with these linear fits ranged from .957 to .993 across all subjects. The associated slopes and intercepts ranged from .863 to 1.05 and from .003 to .045 , respectively. However, a matching analysis compares obtained reinforcement ratios with observed response and time ratios; it cannot be used to predict, in advance, which conditions will produce differences between obtained and programmed rates of

Table 2

Total Time (in sec) Spent Responding On, Total Responses Emitted To, and Total Reinforcers Obtained from Each Component of Each Concurrent Schedule

\begin{tabular}{|c|c|c|c|c|c|c|c|c|}
\hline Subject & $15 \mathrm{sec}$ & $1 \mathrm{~min}$ & $30 \mathrm{sec}$ & $1 \mathrm{~min}$ & $1 \mathrm{~min}$ & $1 \mathrm{~min}$ & $2 \mathrm{~min}$ & $1 \mathrm{~min}$ \\
\hline \multicolumn{9}{|c|}{ Time } \\
\hline 1 & 1328 & 2 & 2536 & 164 & 2196 & 1396 & 787 & 2810 \\
\hline 2 & 1636 & 8 & 1945 & 895 & 1558 & 2036 & 1306 & 2293 \\
\hline 3 & 1410 & 0 & 1788 & 751 & 1716 & 1817 & 1223 & 2376 \\
\hline 4 & 1437 & 87 & 1952 & 614 & 2068 & 1505 & 1435 & 2159 \\
\hline 5 & 1381 & 10 & 2140 & 609 & 1934 & 1661 & 921 & 2671 \\
\hline 6 & 1443 & 11 & 2596 & 31 & 2615 & 979 & 1340 & 2259 \\
\hline \multicolumn{9}{|c|}{ Responses } \\
\hline 1 & 1796 & 1 & 4187 & 175 & 2016 & 1583 & 1204 & 3418 \\
\hline 2 & 916 & 5 & 1453 & 735 & 1011 & 1277 & 1258 & 2022 \\
\hline 3 & 2154 & 0 & 3160 & 1135 & 2443 & 2066 & 1848 & 3369 \\
\hline 4 & 1079 & 57 & 2317 & 722 & 1688 & 1655 & 1497 & 2879 \\
\hline 5 & 688 & 2 & 1546 & 342 & 907 & 794 & 532 & 1486 \\
\hline 6 & 1118 & 2 & 1867 & 30 & 1063 & 907 & 1012 & 2454 \\
\hline \multicolumn{9}{|c|}{ Reinforcers } \\
\hline 1 & 81 & 0 & 78 & 3 & 43 & 30 & 15 & 48 \\
\hline 2 & 81 & 0 & 60 & 21 & 34 & 40 & 20 & 43 \\
\hline 3 & 81 & $\mathbf{0}$ & 60 & 21 & 40 & 40 & 22 & 47 \\
\hline 4 & 79 & 2 & 63 & 18 & 42 & 32 & 21 & 42 \\
\hline 5 & 81 & $\mathbf{0}$ & 66 & 14 & 37 & 35 & 16 & 48 \\
\hline 6 & 81 & 0 & 81 & 0 & 45 & 19 & 19 & 43 \\
\hline
\end{tabular}

Note-All statistics have been averaged over the last five sessions of each schedule; the two presentations of conc VI 1-min VI 1-min have been averaged together. 
reinforcement, or what the specific rates of obtained reinforcement will be.

In contrast, Houston and McNamara (1981) made specific predictions regarding the allocation of behavior under different combinations of $\tau$ and $\lambda$. These predictions are with respect to the division of time between two concurrently available schedules. If the response rates are constant and equal for both alternatives, then time-allocation and response-allocation measures should be proportional. Houston and McNamara made no predictions about actual rates of responding on each of the alternatives but did assume that "the animal's rate of responding is high enough for it to obtain all the rewards as soon as they are set up" (p. 383).

The data in Table 2 show qualitative support for Houston and McNamara's (1981) predictions regarding the animals' time allocation. In the conc VI 15-sec VI 1-min condition, $\tau$ was equal to 1.0 , and all subjects showed nearly exclusive preference for the richer schedule. The proportion of time spent on the richer schedule was $1.00,1.00,1.00, .94, .99$, and .99 for Subjects 1 through 6, respectively. The proportion of responses to the richer schedule showed a similar range. In contrast, in the conc VI 1-min VI 1-min condition, where $\lambda$ was 1.0 and $\tau$ was .25 , the proportion of time spent on one side was $.61, .57, .51, .58, .54$, and .73 for Subjects 1 through 6 , respectively. These proportions are values close to .5 , except for that of Subject 6 . This animal exhibited almost exclusive preference for one alternative during the initial baseline presentation of the conc VI 1-min VI 1-min schedules; during the second presentation of this schedule, performance was not as extreme. Response proportions were comparable for all subjects.

As predicted, in the conc VI 30-sec VI 1-min condition $(\tau=0.50, \lambda=0.50)$ the subjects showed more extreme partitioning of their behavior with respect to the richer schedule than the proportion of programmed reinforcement rates (.67 with respect to the 30 -sec schedule). The proportion of time spent responding to the $30-\mathrm{sec}$ schedule was $.94, .68, .70, .76, .78$, and .99 for the 6 animals. The range of response proportions was similar. In the conc VI 1 -min VI 2-min condition $(\tau=0.25$, $\lambda=0.50$ ) the proportions were not as high. The proportion of time spent on the $1-\mathrm{min}$ schedule was $.78, .64$, $.66, .60, .74$, and .63 for the 6 subjects. The response proportions were similar. Contrasting these two conditions, in which the ratio of rich to lean schedule was the same, it can be noted that in the condition with the smaller value of $\tau$, both response and time proportions were closer to the proportions expected from the programmed reinforcement rates.

The Houston and McNamara (1981) model predicts that the time proportions for the four schedule combinations should be ordered as follows: conc 1-min 1-min, conc 1-min 2-min, conc 30-sec 1-min, and conc 15-sec 1-min. Page's (1963) test for ordered alternatives found that the time proportions differed across the four conditions as predicted $(L=179, m=6, n=4, p<.01)$. Response proportions showed the same pattern $(L=180, m=6$, $n=4, p<.01$ ).

One implication of the optimization model is that performance will optimize the rate of obtained rewards. The model establishes optimal stay times (i.e., time spent on an alternative after the COD has elapsed) for each of the available schedules under varying conditions. These optimal stay times are denoted $a_{1}{ }^{*}$ and $a_{2}{ }^{*}$, where $a_{1}{ }^{*}$ is the optimal stay time on the richer schedule and $a_{2}{ }^{*}$ is the optimal stay time on the leaner schedule. Any deviations from these optimal stay times will result in a reduction in the obtained reward rates.

Houston and McNamara (1981) constructed two measures to use in evaluating actual stay times against optimal stay times. The first of these measures, $R^{*}$, represents the maximum reward rate obtainable for an animal whose stay times correspond to $a_{1}{ }^{*}$ and $a_{2}{ }^{*} . R^{*}$ is the sum of $R_{1}{ }^{*}$ and $R_{2} *$ (where $i=1$ denotes the richer schedule, and $i=2$ denotes the alternative). In the stay-switch region, these optimal values are obtained by solving, numerically, two equations:

$$
R_{1}^{*}=\left[a_{1}^{*}+1-\exp (-2 \tau)\right] /\left(a_{1} *+2 \tau\right)
$$

and

$$
R_{2}{ }^{*}=\left\{1-\exp \left[-\lambda\left(a_{1} *+2 \tau\right)\right]\right\} /\left(a_{1} *+2 \tau\right) .
$$

The second measure, $S^{*}$, represents the number of changeovers per minute that will optimize the reward rate. $S^{*}$ is calculated as follows:

$$
S^{*}=2 /\left(a_{1}^{*}+2 \tau\right) \text {. }
$$

Similar formulas apply in the never switch and stay-stay regions. These measures provide two methods for evaluating performance. First, the obtained reward rate can be compared to that predicted by the optimal strategy. Second, the predicted switch rate, $S$, can be compared to the number of changeovers per minute. The mean observed values of $R_{1}, R_{2}$, and $S$, and the results predicted by the Houston and McNamara (1981) model, are presented in Table 3.

\section{Obtained versus Optimal Reward Rate}

In Figure 1, the obtained reinforcement rate is compared with the optimal rate for each schedule combination. In each case, the animals failed to achieve the levels of reinforcement predicted by the theory. The theoretical optimum is based on a continuous function that has a constant probability of reinforcement. In our paradigm, a discrete approximation to this continuous function was employed; therefore, an animal following the exact strategy specified by Houston and McNamara (1981) might show deviations from optimality due to the endpoint of the reinforcement cycle for any given session. Accordingly, the average reinforcement rate across the five stability sessions was used for statistical analysis, except where noted.

To evaluate the observed deviations from optimality, the ratio of optimal to obtained overall reinforcement rate 
Table 3

Optimal and Observed Values (in units/min) of Switch Rate, Reward Rate on the Richer Schedule, and Reward Rate on the Leaner Schedule

\begin{tabular}{|c|c|c|c|c|}
\hline Subject & $15 / 60$ & $30 / 60$ & $60 / 60$ & $60 / 120$ \\
\hline \multicolumn{5}{|c|}{ Switch Rate } \\
\hline Optimal & 0.00 & 0.78 & 3.33 & 1.81 \\
\hline 1 & 0.05 & 0.54 & 1.16 & 0.92 \\
\hline 2 & 0.10 & 0.86 & 1.06 & 1.37 \\
\hline 3 & 0.02 & 1.23 & 1.48 & 1.47 \\
\hline 4 & 0.45 & 1.15 & 1.60 & 2.08 \\
\hline 5 & 0.14 & 0.63 & 1.13 & 0.84 \\
\hline 6 & 0.05 & 0.02 & 1.09 & 1.39 \\
\hline \multicolumn{5}{|c|}{ Richer Schedule } \\
\hline Optimal & 4.00 & 1.71 & 0.79 & 0.90 \\
\hline 1 & 3.65 & 1.74 & 0.72 & 0.80 \\
\hline 2 & 2.96 & 1.26 & 0.56 & 0.72 \\
\hline 3 & 3.45 & 1.41 & 0.67 & 0.79 \\
\hline 4 & 3.12 & 1.48 & 0.71 & 0.70 \\
\hline 5 & 3.49 & 1.45 & 0.62 & 0.81 \\
\hline 6 & 3.34 & 1.84 & 0.74 & 0.72 \\
\hline \multicolumn{5}{|c|}{ Leaner Schedule } \\
\hline Optimal & 0.00 & 0.56 & 0.79 & 0.38 \\
\hline 1 & 0.00 & 0.06 & 0.50 & 0.25 \\
\hline 2 & 0.00 & 0.45 & 0.67 & 0.33 \\
\hline 3 & 0.00 & 0.50 & 0.68 & 0.36 \\
\hline 4 & 0.07 & 0.41 & 0.54 & 0.36 \\
\hline 5 & 0.01 & 0.31 & 0.58 & 0.27 \\
\hline 6 & 0.00 & 0.01 & 0.31 & 0.32 \\
\hline
\end{tabular}

Note-Observed switch rates were calculated by dividing the total number of switches in a session by the total session length; obtained reward rates were calculated by dividing the total number of reinforcers received from each schedule by the cumulative time spent responding on that schedule within each session. For each subject, the observed switch rate and the obtained reward rates were averaged over the last five sessions the schedule was in effect; the two presentations of conc VI 1-min VI 1-min have been averaged together.

was computed for each subject and for each condition. This ratio provides a measure of relative efficiency under the assumptions of the model. Although relative efficiency was less than 1.00 in all cases, it did not seem to be systematically related to the different values of the alternative schedule. This impression was supported by two types of analyses. First, the mean relative efficiency of each schedule combination was ranked with animals, and this ranking was used to compute a Kendall coefficient of concordance (Siegel, 1956) across animals. The value was not reliable $[W(4,6)=.411, p>.10]$, indicating that, across subjects, one schedule combination was not uniformly low in relative efficiency. Second, to assess efficiency within subjects, the relative efficiency measures obtained from the last 5 days of each condition were regressed on the IRI of the alternately available schedule. In only one case (Subject 2) did the slope of the line significantly differ from zero $[t(23)=3.65, p<.05]$. Therefore, within subjects, relative efficiency tended to be constant across a range of conditions, although across subjects, there was a difference in relative efficiency.

Several authors have noted that large changes in behavior allocation across conc VI VI schedules do not af- fect obtained reinforcement rates (e.g., Heyman, 1983; Staddon \& Motheral, 1978). This argument would imply that relative efficiency might not be sensitive to changes in schedule parameters. However, these observations are based on paradigms that employ a shorter COD than that used in this study. To test if the relative efficiency measure was sensitive to changes in the schedule parameters, simulated sessions were run. The same overall switch rates and session lengths obtained by the 6 animals on each schedule combination were used, but the time allocation across schedules was set at 50:50. This procedure simulates choice behavior that is indifferent to the underlying schedule parameters.

The results of this analysis are presented in Figure 2. It is clear that, under the parameters used in this study, the relative efficiency of an animal that is indifferent changes with the schedule combination. This contrasts with the invariance in relative efficiency evident in the mean from the 6 animals.

\section{Obtained versus Optimal Switch Rate}

The obtained switch rates over the last five sessions of each schedule were analyzed to determine if they differed from the values predicted by the Houston and McNamara (1981) model. The rates were evaluated with respect both to the ordinal predictions derived from the model and to the exact quantitative values.

The model predicts that switch rates should be ordered as follows: conc 15-sec 1-min, conc 30-sec $1 \mathrm{~min}$, conc 1-min 2-min, and conc 1-min 1-min. As can be seen in Table 3, this pattern is present in the mean switch rate across the five stability sessions. Page's (1963) test for ordered alternatives indicated that this ordering was reliable $(L=176, m=6, n=4, p<.01)$.

The mean switch rates were also assessed relative to the values predicted by the Houston and McNamara (1981) model. Because switch rates are based on the counted number of switches within a session, a statistical analysis predicated on a normal distribution was judged inappropriate; rather, the number of switches were assumed to be distributed as a Poisson random variable. Under this distribution, the $95 \%$ confidence intervals for the total number of switches across the last five sessions of each schedule combination were calculated according to a procedure devised by Johnson and Kotz (Shapiro \& Gross, 1981, p. 122).

These intervals are given in Table 4. Comparison of these intervals with the predicted values indicated that all subjects switched more than predicted in the conc VI 15-sec VI 1-min condition. In contrast, all animals underswitched in the conc VI 1-min VI 1-min condition, and all but 1 underswitched in the conc VI 1-min VI 2-min condition. The results were less consistent in the conc VI 30 -sec VI 1-min condition. The underlying pattern appears to be that as the predicted number of switches increases, animals change from overswitching to underswitching of predicted rates. 


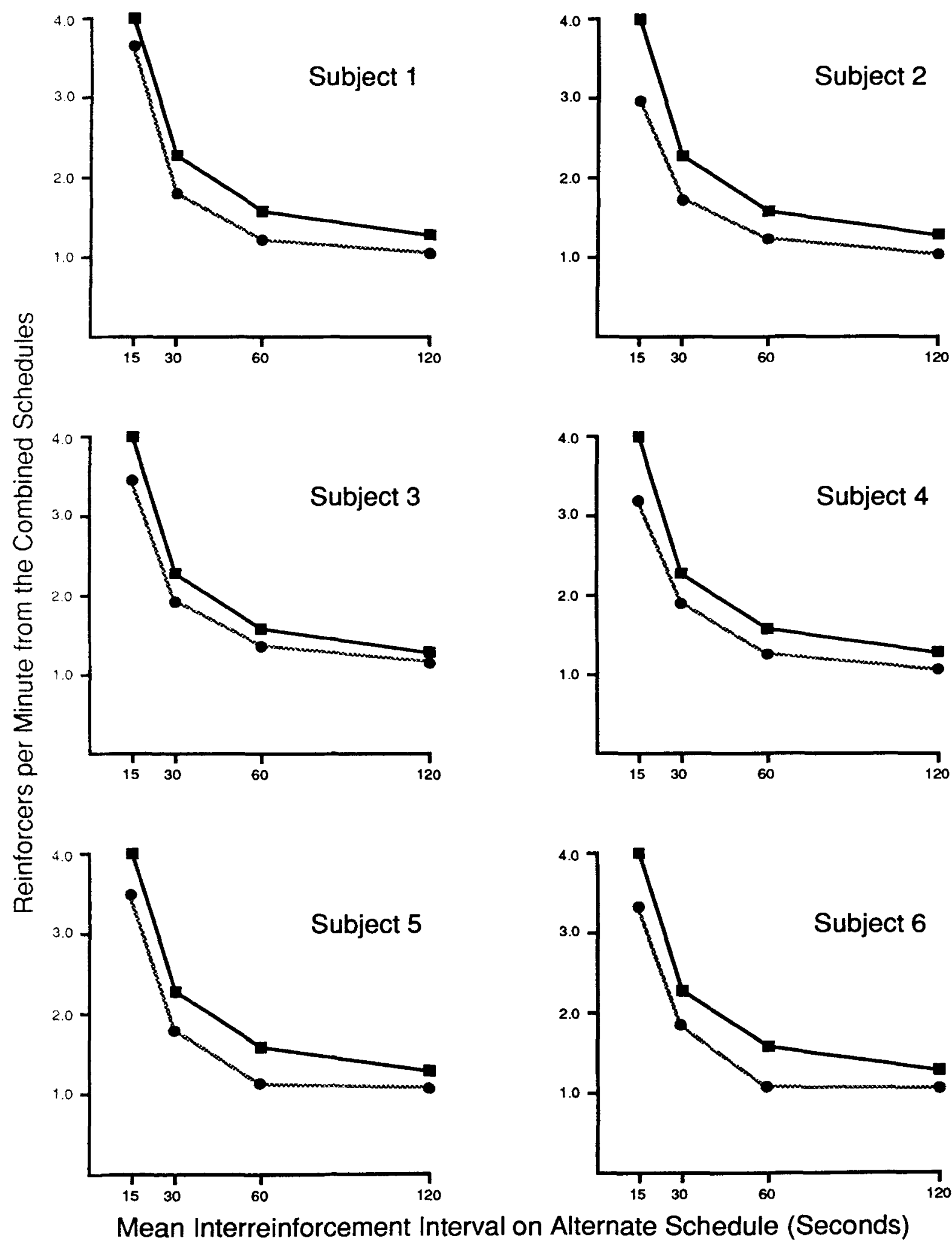

Figure 1. Optimal (a) and obtained (o) reinforcers per minute as a function of the alternately available schedule. For each subject, the obtained reinforcement rate was averaged over the last five sessions for each schedule; the two presentations of conc VI 1-min VI 1-min have been averaged together. 


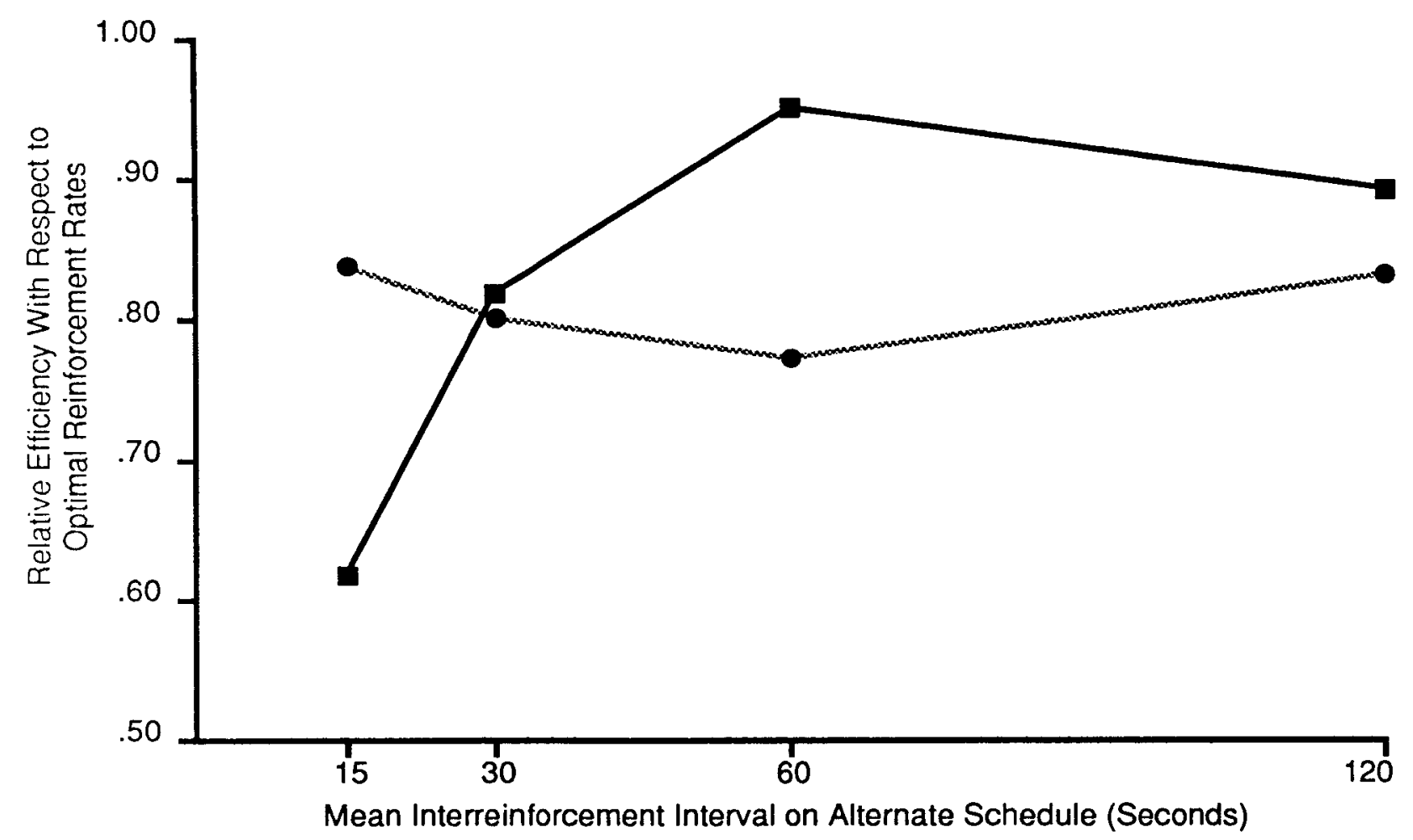

Figure 2. Simulated relative efficiency at indifference ( $(\bullet)$ and observed relative efficiency ( $\bullet$ ) as a function of the average interreinforcement interval on the alternately available schedule. The observed values were calculated by averaging across all subjects over the last five sessions for each schedule pair; the two presentations of conc VI 1-min VI 1-min have been averaged together.

\section{DISCUSSION}

The present results show some support for Houston and McNamara's (1981) model in a conc VI VI procedure using a long COD. Behavioral allocation in the never switch region, in the stay-stay region, and in the stay-switch region was consistent with predictions. Furthermore, within the stay-switch region, the proportion of responses to the richer schedule was higher in the condition with the larger value of $\tau-$ a result predicted by the model. The rate of switching between alternatives also followed the ordering predicted by Houston and McNamara's model.

The results are also consistent with the accounts of conc VI performance provided by Herrnstein's (1961) matching law and by Herrnstein and Vaughan's (1980) melio-

Table 4

Upper and Lower Bounds of the Ninety-five Percent Confidence Intervals of Switch Rates (per min) for Each Schedule Combination

\begin{tabular}{ccccc}
\hline & \multicolumn{4}{c}{ Alternate Schedule Value } \\
\cline { 2 - 5 } Subject & $15 \mathrm{sec}$ & $30 \mathrm{sec}$ & $2 \mathrm{~min}$ & $1 \mathrm{~min}$ \\
\hline 1 & $0.01-0.11$ & $0.46-0.66$ & $1.08-1.25$ & $0.82-1.04$ \\
2 & $0.06-0.17$ & $0.74-0.98$ & $0.97-1.14$ & $1.24-1.51$ \\
3 & $0.00-0.06$ & $1.09-1.39$ & $1.32-1.56$ & $1.34-1.62$ \\
4 & $0.34-0.58$ & $1.02-1.31$ & $1.50-1.71$ & $1.92-2.25$ \\
5 & $0.08-0.22$ & $0.53-0.74$ & $1.04-1.22$ & $0.74-0.95$ \\
6 & $0.02-0.12$ & $0.01-0.05$ & $1.01-1.18$ & $1.26-1.53$ \\
\hline
\end{tabular}

ration model. That is, both of these models would allow for the outcomes observed here (see, e.g., Boelens, Kop, Nagel, \& Slangen, 1987). However, it should be noted that the observed outcomes are only part of a large outcome space predicted by these two models. The matching law predicts that behavioral allocation will match the ratio of observed rates of reinforcement. It does not predict the separate levels of behavior themselves or the conditions under which the observed rate of reinforcement will differ from the programmed rate (Houston \& McNamara, 1981, p. 381).

Similarly, the results obtained here are only one of many outcomes consistent with the melioration model. For example, in the conc VI 15-sec VI 1-min condition, 5 of the 6 animals spent $99 \%-100 \%$ of their time on the richer alternative and produced switch rates below .15 per minute. This pattern of behavior approximates exclusive preference with a switch rate of zero, and is consistent with Equation 2. However, consider the local rate of reinforcement with a different pattern of allocation. After $5 \mathrm{~min}$ of responding on the VI 15-sec schedule, a response to the other alternative has a .995 probability of being reinforced if the animal switches to that alternative and waits until the end of the COD. Therefore, by switching to the 1-min alternative, collecting the reinforcer, and returning to the 15 -sec alternative, the animal can obtain a rate of 4 reinforcers per minute on both alternatives. 
This pattern would also satisfy the melioration assumption but would produce a preference of only $95 \%$ and a switch rate of .38 per minute.

The general point is that the melioration model does not make a unique prediction concerning the behavioral allocation and the ordering of switch rates in this experiment. Both the melioration model and the matching law specify the relationship between the behavioral outcomes (such as the responses the two alternatives or the obtained rates of reinforcement) on a given concurrent manipulation. In this sense, the two models specify what Spence (1948) termed "R-R laws" of behavior. The Houston and McNamara (1981) model, in contrast, develops specific predictions of stay times and switch rates that are tied to the independent variables of the underlying schedules.

The monotonic ordering of both reward rates and switch rates predicted by Houston and McNamara's (1981) model was evident in the observed behavior. However, overall reward rates were less than the optimal rate, which implies that the animals were not completely efficient under the assumptions of the model. The results of the linear regression tests indicated that relative efficiency was about $80 \%$ and did not vary generally with different schedule values. This invariance contrasts sharply with the relative efficiency that was obtained when a time allocation based on schedule indifference was simulated. In the latter case, observed efficiency was a function of the programmed schedules. Heyman (1983) has suggested that "systematic covariation, even with large absolute deviations between predictions and results, would suggest that animals are in some sense maximizers"' (pp. 18). In a similar vein, we suggest that animals are sensitive to optimality constraints, but are imperfect at implementing an optimal strategy.

There may be many reasons why animals fail to implement the optimal behavioral allocation. For example, the ability of an animal to switch after a set, constant stay time may be imperfect. It may also be that animals are optimizing some factor other than reward rate. Houston and McNamara (1981) noted that their model assumes that time on a schedule is equivalent to time spent switching with respect to the "currency" being maximized. They suggested that

this equivalence is likely to hold for performance in a Skinner box, but not for traveling between patches in the wild. In general, if switching is energetically more costly than staying, the stay times that maximize net rate of energy intake will be longer than those that maximize reward rate (p. 388)

It is possible that the equivalence mentioned by Houston and McNamara (1981) may not hold, even within the operant chamber. In our experiment, response alternatives were spatially separated; when conditions required a high rate of switching to maximize the overall reward rate, switch rates fell below those predicted by the model. These lower switch rates served to reduce the number of reinforcers the animal obtained.
If this latter interpretation is correct, it suggests that the obtained reward rates are "suboptimal" only in the sense that the animal is maximizing some other currency, such as net energy gain. This conclusion must be treated with some caution. The practice of defining the currency in terms of the optimal solution is generally recognized as tautological. It would therefore seem appropriate to examine the energetic costs of the changeover procedure directly before incorporating them formally into the Houston and McNamara (1981) model.

This reasoning also does not explain the inefficiency of animals in the conc VI 15-sec VI 1-min condition. Optimal responding in this condition is predicated on a never switch strategy; hence, the lower efficiency cannot be attributed to a lower rate of switching. It may be that animals also incorporate sampling strategies that interfere with the optimal behavior in a static situation but allow them to respond to changes in environmental contingencies (e.g., Cooper, 1987).

\section{REFERENCES}

Allison, T. S., \& LLoyd, K. E. (1971). Concurrent schedules of reinforcement: Effects of gradual and abrupt increases in changeover delay. Journal of the Experimental Analysis of Behavior, 16, 67-73.

BaUM, W. M. (1974). On two types of deviation from the matching law: Bias and undermatching. Journal of the Experimental Analysis of Behavior, 22, 231-242.

BAUM, W. M. (1979). Matching, undermatching and overmatching in studies of choice. Journal of the Experimental Analysis of Behavior, 32, 269-281.

Boelens, H., Kop, P. F. M., Nagel, A. L., \& Slangen, J. L. (1987). Concurrent schedules: Effects of reinforcement rate and changeover delay on time allocation in a three-alternative procedure. Quarterly Journal of Experimental Psychology, 39B, 229-244.

Catania, A. C., ReYnolds, G. S. (1968). A quantitative analysis of the responding maintained by interval schedules of reinforcement. Journal of the Experimental Analysis of Behavior, 11, 327-383.

COOPER, W. S. (1987). Decision theory as a branch of evolutionary theory: A biological derivation of the Savage axioms. Psychological Review, 94, 395-411.

DE VILLIERs, P. (1977). Choice in concurrent schedules and a quantitative formulation of the law of effect. In W. K. Honig \& J. E. R. Staddon (Eds.), Handbook of operant behavior (pp. 233-287). Englewood Cliffs, NJ: Prentice-Hall.

Findley, J. D. (1958). Preference and switching under concurrent scheduling. Journal of the Experimental Analysis of Behavior, 1, 123-144.

HERRNSTEIN, R. J. (1961). Relative and absolute strength of response as a function of frequency of reinforcement. Journal of the Experimental Analysis of Behavior, 4, 267-272.

Herrnstein, R. J., \& VAUGHAN, W., JR. (1980). Melioration and behavioral allocation. In J. E. R. Staddon (Ed.), Limits to action: The allocation of individual behavior (pp. 143-176). New York: Academic Press.

Heyman, G. M. (1983). Optimization theory: Close but no cigar. Behaviour Analysis Letters, 3, 17-26.

Houston, A. I., \& McNamara, J. (1981). How to maximize reward rate on two variable-interval paradigms. Journal of the Experimental Analysis of Behavior, 35, 367-396.

Norman, W. D., \& MCSweeney, F. K. (1978). Matching, contrast, and equalizing in the concurrent lever-press responding of rats. Journal of the Experimental Analysis of Behavior, 29, 453-462.

PAGE, E. B. (1963). Ordered hypotheses for multiple treatments: A significance test for linear ranks. Journal of the American Statistical Association, 58, 216-230. 
SHAPIRo, S. S., \& Gross, A. J. (1981). Statistical modeling techniques. New York: Marcel Drekker.

SIEGEL, S. (1956). Nonparametric statistics for the behavioral sciences. New York: McGraw Hill.

SPENCE, K. W. (1948). The methods and postulates of "behaviorism." Psychological Review, 55, 67-78.

Staddon, J. E. R., \& Motheral, S. (1978). On matching and maximizing in operant choice experiments. Psychological Review, 85, 436-444.
Stevens, S. S. (1957). On the psychophysical law. Psychological Review, 64, 153-181.

WEARDEN, J. H., \& BuRGESS, I. S. (1982). Matching since Baum (1979). Journal of the Experimental Analysis of Behavior, 38, 339-348.

(Manuscript received July 19, 1988; revision accepted for publication January 6,1989 .)

\title{
Announcement
}

\author{
30th Annual Meeting of the Psychonomic Society \\ Atlanta, Georgia \\ November 17-19, 1989
}

The 30th Annual Meeting of the Psychonomic Society will be held in Atlanta, Georgia, November 1719, 1989. The meetings will begin Friday morning and continue until Sunday at noon. The headquarters hotel will be the Hyatt Regency.

The program and hotel reservation cards will be mailed to members and associates in September. A copy of the program will be published in the November issue of the Bulletin of the Psychonomic Society.

For further information, please contact the secretary-treasurer of the Society: Michael E. Rashotte, Department of Psychology, Florida State University, Tallahassee, FL 32306-1051 (Telephone: 904-644-2040; BITNET: Rashot@FSU). 